\title{
Integrating Information Systems
}

\section{A Semiotics Approach}

\author{
Valerie A. Martin, Mark Lycett and Robert Macredie \\ Department of Information Systems and Computing, Brunel University, Uxbridge, Middlesex, \\ UB8 3PH, Phone: 01895754000 Fax: 01895 251686, email: \\ \{valerie.martin|mark.lycett|robert.macredie\}@brunel.ac.uk
}

\begin{abstract}
Businesses often have heterogeneous information systems which have diverse 'meanings' embedded in them. Consequently, systems have difficulties in talking to each other, and users cannot interact with them effectively. There are semantic barriers which inhibit a seamless flow of information and the ability to reuse knowledge. As a result, software developers often cannot model and capture the 'messiness' of diverse mental models and language, which are a normal part of organisational life. This situation highlights the need to integrate people and technology in a way which can enable a shared, conceptual understanding within and between the cultures in which the systems are established. Successful integration should allow both systems and the organisation to adapt to change.

This paper reviews the varied and often conflicting paradigms in information systems integration, with regard to the issue of multiple meanings. With this in mind, the authors develop an integrated framework to investigating these issues within the scope of organisational semiotics.
\end{abstract}

Key words: Information systems integration, semantics, meaning, language, semiotics

\section{INTRODUCTION}

A typical organisation uses an historical accumulation of heterogeneous information systems. Each system implicitly embody a plethora of complex business practices, structures, rules and procedures. These systems also embody terms, concepts and objects which represent the real world. In each of these systems there is much embedded 'meaning', rooted in the particular domain and culture the system has been developed for. These meanings

The original version of this chapter was revised: The copyright line was incorrect. This has been corrected. The Erratum to this chapter is available at DOI: 10.1007/978-0-387-35611-2_22 
quickly become outdated and often conflict with the meaning embedded in other systems.

This situation is proving to be a major difficulty in today's global business environment, where it is increasingly necessary to enable systems to talk to each other, both internally and with customers and other stakeholders. For example, mergers and acquisitions, process improvement, web technology and customer relationship management are ways in which businesses are becoming more integrated with each other and their customers. Users should be able to work with software effectively without having to understand the underlying technology. Consequently, there is a need to come to a shared conceptual understanding on the aspects of the business which need to be modelled in a way which can allow both people and technology to work together in harmony and adapt to change.

This paper explores the issue of multiple meanings in relation to systems integration, and explains how an approach from organisational semiotics can provide a conceptual framework to investigate the problem areas. Section 2 presents the problem of human and software semantic barriers. Section 3 reviews the prevalent approaches to the integration of people, processes and technology. Section 4 introduces organisational semiotics and illustrates how this can provide a 'framework for analysis' for systems integration and the problem of semantics. Section 5 concludes that semiotics can provide clarification of 'meaning' for information systems integration.

\section{THE SEMANTIC BARRIER}

\subsection{Multiple Meanings and Language}

There are semantic barriers to systems integration. Multiple meanings have to be represented in software in a way which can be understood by both people and software. Meaning in software is made explicit through language, through such tools as modelling languages, programming languages and ontology languages. Language can incorporate text, mathematical formulas, sound, diagrams, and even natural language. The meaning with regard to language representation is often referred to as 'semantics'. With people multiple meanings are usually highly tacit, although they can be made explicit through the use of language, either spoken or in text. 


\subsection{Organisational Semantics}

In spite of this, some recent work has pointed out the need to recognise the issue of organisational semantics. Firstly, there is the issue of 'tacit' multiple meanings. Tenkasi and Boland (1998) claim that the work of Wittgenstein on 'Semantic Mediational Theories' is the basis of knowledge sharing in organisations. They point out that knowledge cannot be shared effectively unless people have the capability to understand the different perceptions of others. Secondly, with regard to working with information systems, people often have different usages of language, or simply different cultural interpretations. Hasselbring (2000) claims systems integration can be impeded through human semantic barriers in the form of homonyms (the same concepts, terms, and words can be interpreted in different ways) and synonyms (different concepts, terms and words can be given a similar meaning).

\subsection{Software Issues}

Ontologies, process models, databases, knowledge repositories, intranets, groupware, the internet are software-based tools which represent and model the business. They can have semantic problems in terms of integration with other systems and with people. The semantic 'messiness' which is so often a normal part of organisational life is difficult to capture in software. Software needs to be developed in a way which can allow users to work with the language and concepts they are used to. At the same time language must be standardised to some degree in software in a way which can provide some common basis for communication. At present software cannot talk to each other, the models embedded are diverse, often are not compatible and do not reflect the business and the users' needs (de Cesare et al. 2001, Chandrasekaran et al. 1998)

\section{$2.4 \quad$ Semantic Gaps}

These semantic barriers reveal a gap between the developers of systems and the 'real' world of the organisation. Developers need to know what to model in order to manifest a shared conceptual understanding in the systems. In terms of systems integration, they need to understand not only how to represent and manipulate information, but also how users mental models are expressed through the content and the use of language. Defining and formalising the different levels of a 'shared conceptual understanding' and what it represents within the changing business context is a major challenge facing systems integrators. This knowledge has to be defined and embedded 
in a flexible manner in the software, using appropriate tools, languages and architectures.

\section{MULTIPLE MEANINGS AND INFORMATION SYSTEMS INTEGRATION}

\subsection{Organisational Approaches}

The problem of diverse mental models and meanings has long been recognised in relation to information systems. Soft Systems Methodologies and Socio-technical Approaches are methods to unearth mental models while enabling people to learn. They allow information systems developers to gain a clearer understanding of user requirements and tacit mental models and therefore reach some consensus on how the information systems can accommodate these multiple meanings (Checkland 1981, Mumford 1994). Also, some cultural approaches to information systems integration have recognised the need to share a 'common understanding' of information and business concepts. Davenport (1993), for example, suggests a contention between information globalism and information particularism. All organisations have a need for very generic terms, as well as a need for more specific terms for various functions and sub-cultures. However, these paradigms are inadequate in many respects; especially they do not give much idea on how systems developers can go about representing the details of these diverse understandings and meanings through software.

Nonaka and Tageuchi (1995) appreciate that diverse mental models can be made explicit and shared, through their theory of tacit and explicit knowledge. Tacit knowledge, or mental models, resides in people's heads, whereas explicit knowledge can take the form of dialogue, text or computer software. Mental models are made explicit and can be transferred easily explicit to explicit, and can then be embedded back into tacit knowledge again. These ideas give little insight on semantic mapping - how this knowledge can be specified and modelled by systems developers, especially in relation to systems integration.

Orlikowski (1994) developed an approach to information technology, based on Structuration Theory and the work of Giddens (1984). The two opposing views of technology, determinist and social constructivist, are recognised in that both technology and organisation are equally important and interdependent. Structuration Theory incorporates the perspectives of different people regarding technology within the dynamic structure of the organisation and realises the benefits in viewing the interdependence as an 
ongoing process of change. It also recognises diverse mental models and meanings, though, again, there is little attempt to define how these diverse meanings can be made explicit and mapped by software developers.

More emphasis on meaning in relation to language is made through the Language Action Perspective (LAP). Winograd (1987) claims that the Language Action Perspective sees people as linguistic beings who act through language. They recognise the difficulty of representing and mapping the semantics, that is, how the systems designers make the tacit mental models of people explicit, especially expressed through language. Again, however, they give little indication on how to do this, and they focus more on the content and use of language, rather than its representation. There is simply not enough detail for software developers to be able to work with.

\subsection{Technological Approaches}

There are software tools, approaches, and techniques which can be used to make diverse mental models and meanings more explicit. Formal ontologies are designed to enable communication between computer systems and people through explicitly defining the meaning of terms and concepts from the user's perspectives, and they can range from being highly structured through rigorous specification of terms, to highly informal, expressed in natural language (Uschold 1996). They are technically strong and they are genuine attempts to capture the content of language and mental models. The extent to which systems designers can be sure of a consensus on user perspectives being reached is questionable, however. They would have ensure that mental models are understood and some sort of consensus being reached before embedding the different representations into the architectures.

The Unified Modelling Language (UML) provides explicit models of the business from different perspectives. The UML provides support for extending the language for defining domain-specific meta-elements (de Cesare 2001). Again, the question is the extent to which the modellers can be sure of a consensus being reached on mental models, that is, the models may be the view of users or they may simply be the views of the designers. Component-Based Software Development (CBSD) focuses on standardising the interfaces between autonomously developed building blocks of software (Caron 1999). The idea is to integrate heterogeneous software using standards for message-passing, although little work has been done on how to represent meanings in software components so that they can become interoperable. Again, there is the issue of the extent to which systems designers understand the needs of the business and the users. The above 
approaches place emphasis on the structure of representation or the syntactic aspect of the models.

\subsection{Language Content, Use and Representation}

The organisational approaches described above recognise to an extent the issue of mental models and meanings, especially in regard to information systems. Unfortunately, there is not enough detail to allow a refined mapping and specification for software developers, especially in relation to systems integration. The approaches tend to focus on the content and the use of information and language, but not the representation. Software tools are common ways of modelling business processes and concepts. They are technically strong, but give no guarantee that the concepts modelled are truly representative of the diverse mental models of the users, especially in messy organisational settings. They tend to focus on the representation, or syntax of language, but not the content or use. Systems integration in this sense is about semantic integration. Semantic integration is about all of these aspects - the content, use and representation of language.

\section{ORGANISATIONAL SEMIOTICS}

\subsection{Semiotics and Language}

An exploratory conceptual framework for the analysis of semantic integration may be provided through the evolving area of organisational semiotics. Semiotics is the process of analysing signs and how they function. Stamper (1988) claims that 'meaning' is the information conveyed between a sign and reality. He points out that organisational semiotics takes both the human and social aspects of information and technological aspects into consideration.

Liu (2000) argues that a major problem with information systems development is that users cannot check on the meanings of words, even with the help of data dictionaries, and analysts may wrongly understand user requirements. A method with an emphasis on semantics is needed, to clarify meanings. Expressed through actions, the world can be created and changed through language. It is therefore important, he says, to view language as an active means of construction of a world. Lyytinen (1987) points out that language as a purely descriptive, representational phenomenon is not suitable for studying problems of information systems in social settings. Similarly, Heusden and Jorna (1999) argue that the language paradigm in 
general semiotics takes the existence of signs (language) for granted. The paradigm views language as a passive manifestation of the organisation, rather than something which is an interactive and dynamic part of organisational life. Liu views organisational semiotics as a way to bring language to life, and he illustrates the concepts through the semiotic ladder, based on Stamper (1973). Around this the authors of this paper have placed the various organisational and software-based approaches discussed in Section 3, and the semantic gap is illustrated through the question marks (See Figure 1). The semiotic ladder provides a multi-level, multi-disciplinary perspective on people and technology in their contextual setting. This diagram is not an attempt to put these well-established theories and tools into any hierarchical order, but to simply illustrate that there is a semantic gap between organisation and software.

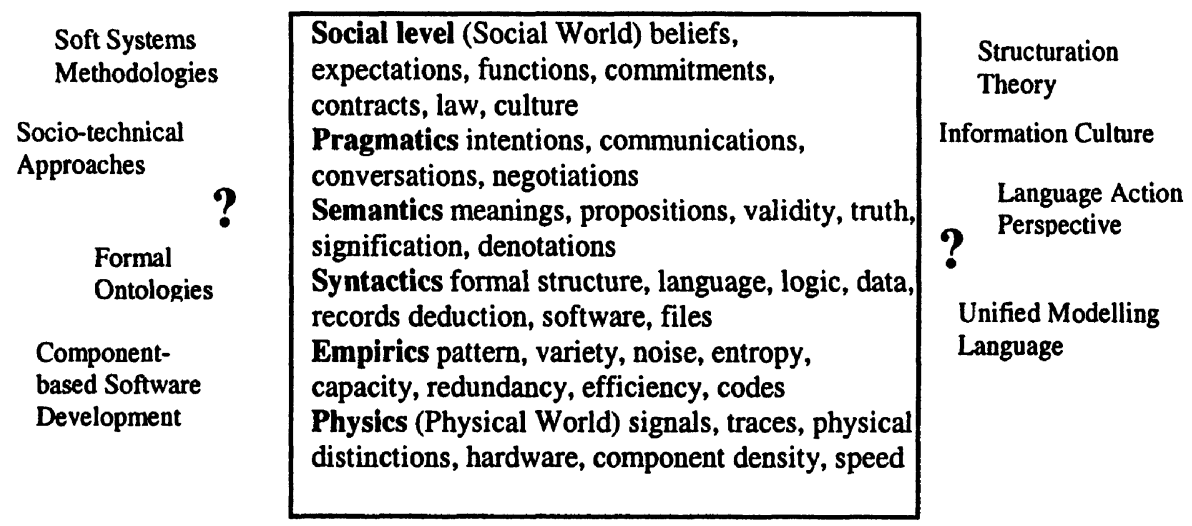

Figure 1. Systems Integration from a Semiotics Perspective (Adapted from Stamper 1973)

\subsection{Semiotics and Systems Integration: an Agenda for Research}

Integrating information systems embedded with diverse meanings is a highly important research agenda, and one which has little clarity. The area of language with regard to both people and software is overlooked and misunderstood. The linguistic term 'semantics' in particular is ambiguous. How systems are integrated through a clearer understanding of both organisational and software semantics is relevant to the complex activities and structures of organisational life.

The approaches outlined in Section 3 indicate the organisational issues being concentrated in the upper levels of the semiotic ladder, and the technological approaches in the lower levels. In the upper levels the focus is on the content and use of language, and in the lower levels it is on the representation and structure of language. The question marks represent a 
noticeable lack of clarity at the semantic level, the link between the organisation and the software.

Research using organisational semiotics may well provide insight into the semantic issues. Although it is only in its conceptual stages at present, the semiotic ladder covers a broad and highly multi-disciplinary perspective on organisations. Its advantages are that it can look at any aspect or detail of language and multiple meanings within its context. It can reveal links between semantics, the organisational context and the information system, or systems. It can focus down on specific details or it can provide a metaframework for the study of multiple organisations and systems integration. It is important to recognise that this ladder is a reductionist rather than a systemic perspective, and draws sharp lines between each of the six levels. In reality, semiotics is highly systemic. Semantics in relation to software development and user requirements may closely involve pragmatics and syntactics, while also incorporating aspects of the social level, empirics and physics.

The main areas of research are:

- Semantic mapping: what are the important issues concerning organisational and software semantics, and how are they linked?

- Which specific areas of literature from the broad areas of systems integration and meaning are most useful to the study of semantics?

- How can organisational semiotics enable a clearer understanding of semantic integration?

- How can software developers and users work together to enable semantic integration which can adapt to complex and dynamic business requirements?

\section{CONCLUSIONS}

Systems integration is multi-disciplinary, and requires meaning used in business concepts and processes to be clarified. The issue of integration appears to be based on the reconciliation of traditional 'positivist' paradigms to software development with that of 'interpretive' paradigms to information systems. Much emphasis has been put in this paper to viewing the organisation as a language system involving multiple meanings, both tacit and explicit. Integrating information systems through meaning is referred to as semantic integration.

The investigation of systems integration can be undertaken through organisational semiotics. Semiotics provides a contingency framework for studying semantic integration - thr one which is flexible enough to adopt and adapt methods from a multitude of paradigms and practices, and which 
allows a focus on details as necessary. Such an approach enables a multi level perspective of the organisation, one which incorporates the most salient aspects of the above approaches and methods in a multi-disciplinary framework. The authors suggest that organisational semiotics may allow business processes to be modelled and concepts and terms associated with them to be made transparent to integrate people and technology in adaptive and flexible processes.

\section{REFERENCES}

Caron, J. (1999) Component-Based Software for Scientific Application Development, in 15th International Conference on Interactive Information and Processing Systems for Meteorology, Oceanography, and Hydrology, American Meteorological Society, Jan. 1999, USA.

Cesare S. de, Lycett M. and Patel, D. (2001) Business Modeling with UML: Distilling Directions for Future Research, in Proceedings of OOPSLA Workshop 200, Business Object Design and Implementation IV, Minneapolis, USA.

Chandrasekaran, B., Josephson, J. R. and Benjamins, V. R. (1998) An Ontology of Tasks and Methods, in Proceedings of KAW'98, Eleventh Workshop on Knowledge Acquisition, Modeling and Management, Banff, Alberta, Canada, 18th - 23rd April, http://ksi.cpsc.ucalgary.ca/KAW/KAW98/chandra/

Checkland, P. (1981) Systems Thinking, Systems Practice, Wiley, Chichester, UK.

Davenport, T. (1994) Saving ITs Soul - Human Centered Information Management, Harvard Business Review, 72 (2), 119 - 131.

Giddens, A. (1984) The Constitution of Society: outline of the theory of structuration, Cambridge: Polity Press, 1984; Berkeley: University of California Press.

Hasselbring, W. (2000) Information System Integration, Communications of the ACM, 43 (6), $32-39$.

Heusden, B. van, and Jorna R. (2001) Toward a Semiotic Theory of Cognitive Dynamics in Organisations in Liu, K., Clarke, R.J., Andersen, P.B., Stamper, R.K. (2001) Information, Organisation and Technology: Studies in Organisational Semiotics, Information and Organization Design Series, Volume 1, Kluwer Academic Publishers, Boston, USA.

Liu, K. (2000) Semiotics in Information Systems Engineering, Cambridge University Press, Cambridge, UK.

Lyytinen, K. (1987) Two Views of Information Modeling; Information and Management, 12 (1), 9 - 19.

Mumford, E. (1994) New Treatment or Old Remedies: Is Business Process Reengineering Really Socio-Technical Design?, Journal of Strategic Information Systems, 3 (4), 313 326.

Nonaka, I., and Takeuchi, H. (1995) The Knowledge Creating Company - How Japanese Companies Create the Dynamics of Innovation, Oxford University Press, New York, USA.

Orlikowski, W. (1992) The Duality of Technology: Rethinking the Concept of Technology in Organizations, Organization Science, 3 (3), 398 - 427.

Stamper, R. K. (1973) Information in Business And Administrative Systems, London : Batsford, UK. 
Stamper, R.K. (2001) Organisational Semiotics: Informatics without the Computer?, in Liu, K., Clarke, R.J., Andersen, P.B., Stamper, R.K. (2001) Information, Organisation and Technology: Studies in Organisational Semiotics, Information and Organization Design Series, Volume 1, Kluwer Academic Publishers, Boston, USA.

Tenkasi, R.V. and Boland, R.J. Jr. (1998). Exploring Knowledge Diversity in Knowledge Intensive Firms: a New Role for Information Systems, Journal of Systemic Knowledge Management, I (4), http://www.free-press.com/journals/knowledge/issue1/article4.html.

Uschold, M. (1996) Building Ontologies: Towards a Unified Methodology, in Expert Systems '96, the Sixteenth Annual Conference of the British Computer Society Specialist Group on Expert Systems, 16th - 18th December , Cambridge, UK.

Winograd, T. (1987) A Language/Action Perspective on the Design of Cooperative Work, Human-Computer Interaction, 3 (1), (1987-88), 3- 30.

Wittgenstein, L. (1922/1961) Tractatus Logico-philosophicus, Routledge \& Kegan Paul, London (first English edition with a translation, 1922). 